\title{
A REAL-TIME DRONE MAPPING PLATFORM FOR MARINE SURVEILLANCE
}

\author{
Ilseo Jeon ${ }^{1}$, Sangwoo Ham ${ }^{1}$, Jangwoo Cheon ${ }^{1}$, Anna Maria Klimkowska², Hwiyoung Kim ${ }^{1}$, Kyoungah Choi ${ }^{3}$, Impyeong Lee ${ }^{1, *}$ \\ ${ }^{1}$ Dept. of Geoinformatics, University of Seoul, Seoul, Republic of Korea - (uosKR14, f100pilot, khai0614, huddy, iplee)@uos.ac.kr \\ ${ }^{2}$ Dept. of Geoinformatics, University of Seoul, Seoul, Republic of Korea - anna.maria.klimkowska@ gmail.com \\ ${ }^{3}$ Innovation Growth Headquaters, Korea Agency for Infrastructure Technology Advancement, Gyeonggi-do, Republic of Korea- \\ shale8282@kaia.re.kr
}

Commission I, ICWG I/II

KEY WORDS: UAV, Marine Surveillance, Image Processing, Real-Time Mapping, Ship Detection,

\begin{abstract}
:
Marine incidents have caused serious casualties and damaged on property, and situational awareness and actions are needed to reduce further extensive damage for marine surveillance. The importance of an attempt for maritime monitoring using UAV has been raised, and a platform should be prepared to respond immediately to urgent situations. In this research, a real-time drone image mapping platform is proposed for marine surveillance that receives marine images acquired and transmitted by drones and processes them in real time. The platform proposed in this study is divided into 1) UAV System, 2) Real-time image processing, 3) Visualization. UAV system transfers data from a drone to the ground stations. Real-time image processing module generates individual orthophotos followed by directly georeferencing in real time and detecting ships on the orthophotos. Visualization module enables to visualize the orthophotos. The overall mapping time of 3.26 seconds on average was verified for processing image mapping, and ship detection time for a single image was estimated to be within about 1 second, which corresponds to an environment in which an emergency must be handled. In conclusion, a real-time drone mapping platform that is introduced in this study can be evaluated as being available for maritime monitoring that requires swift responses.
\end{abstract}

\section{INTRODUCTION}

Marine accidents inevitably result in as much property loss and human casualties as natural disasters, and it is not easy to quickly obtain and cope with information about the specific location in which the accident occurs because it happens in the marine environment in one case.

EMSA (European Maritime Safety Agency)'s Annual Overview of Marine Casualties and Incidents 2018 reported 20,616 cases of marine accidents over eight years from 2011 to 2017, with 7,495 casualties and fatalities. According to AGCS (Allianz Global Corporate \& Specialty)' Safety and Shifting Review 2018, the marine insurance claims from 2008 to 2017 were recorded losses of $\$ 1.6 \mathrm{bn}$ from human error alone and $\$ 23.5 \mathrm{bn}$ from the pollution of fish farms or even pirate fishing.

In Korea, geographically surrounded by the sea, maritime space has a large portion of the national industry as well as its function as a channel. 99.7 percent of international trade was carried out by sea, accounting for 11.5 percent of the world's maritime trade, earning \$28.7 billion annually as well as collecting about 7 trillion won in fisheries production as of 2010 (Lee, 2016). On the other side, according to statistics from the Ministry of Maritime Affairs and Fisheries, there have been 14,603 maritime accidents reported in the past nine years, 3,296 human casualties, and more than 2,000 illegal operations have occurred each year, resulting in sustained economic losses. Total property damage from maritime accidents was estimated to reach about $\$ 2.4$ trillion and average $\$ 486$ billion a year. Lee (2016) noted the

\footnotetext{
* Corresponding author
}

need for a system for maritime surveillance to reduce such accidents and economic losses.

Previous studies have been conducted on systems that can remotely monitor the oceans, mainly by satellites, and Carpenter (2013) have insisted that satellites are useful under the control of LRIT (Long-Range Identification and Tracking of Ships) and SSN (Safe Sea Net) to monitor and track the oceans. However, the satellite has difficulty in dealing with critical situations in real time due to time resolution and limitations on its accessibility to pinpoint the exact location. The use of drones as a new platform for real-time monitoring on the sea (ACGS, 2018) has been mentioned that drones could enable faster, more informed decision- making on board, which reduces the impact of incidents. Earlier studies have proved the usability of drones. For the characteristics of flexibility, timeliness, low cost, low consumption, low risk, strong monitor capability and widespread coverage of the UAV, it is very suitable for the missions related to maritime surveillance such as investigation and evidence collection, emergency response, maritime search and rescue, pollution of ship discharge (Duan \& Zhang, 2014).

Dimitriou (2013) simulated the effects of UAVs using the extremely complex maritime environment of the Aegean Sea to explore the effectiveness of UAVs in those conditions. It was found that by integrating one or two UAVs into a traditional surveillance system, it becomes more efficient in the detection and persistent surveillance of enemies and neutral targets. However, it has not been advanced to a real-time marine monitoring system which provides more useful information such as images with accurate locations of automatically detected ships. 
Modern mapping techniques have evolved in terms of accuracy and processing time. Furthermore, technology to automatically detect objects has been developed, and various researches have been carried out on ship detection using data from space borne platforms such as SAR (Synthetic Aperture Radar) in marine surveillance. Using AIS (Automatic Identification System) with satellites have been carried out, but it limits for ships equipped with AIS transceiver devices. In addition, in the case of the use of satellites and marine radars, the accuracy of location of detected ships is relatively low. The reason of coverage of a wide range and a lack of timeliness is due to the fixed amount of time to acquire images. The preceding methods are considerably far away to form a platform that can help solve critical situations faster.

Therefore, a platform that can compensate for these problems is needed, and modules for efficient marine monitoring should be reconsidered together. We suggest a solution combining two technologies mentioned before, to obtain the 'real-time marine monitoring platform using drones' necessary to solve the critical and urgent maritime situation in a short period of time. First, realtime mapping allows image mapping and coordinate extraction to inform the urgent situation in real time. Second, the technology of object detection detects where the actual situation takes place.

\section{METHODOLOGY}

\subsection{Real-Time Drone Mapping Platform}

The real-time drone monitoring platform proposed in this study visualizes the information needed for marine situations in real time over three steps, including UAV System, real-time mapping, and visualization, as seen in Figure 1. 1) UAV System: acquired images are transferred through united a drone with sensors. 2) Real-time image processing includes direct georeferencing which can be performed quickly with sensors data, by images sent to the image processing server to generate individual orthophotos and perform ship detection. An Image processing server is between UAV system and visualization with functions which transmit images and processed images. 3) Finally, the real-time drone mapping platform visualize orthophotos including the location of images and detected ships to understand and analyse the situation.

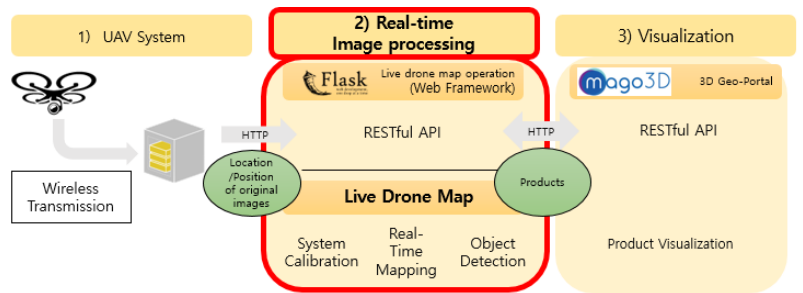

Figure 1. The architecture of real-time platform process diagram for marine surveillance

UAV system: Setting drones with sensors for the data acquisition is the first element which could be an important factor to affect the quality of a range of monitoring, communication, the number of flights depending on the possible flight time for a marine scenario. UAVs are mainly classified into two types: Fixed-wing UAV and Rotary-wing UAV. Fixed-wing UAV has a simple structure whose characteristics allow longer flight duration, higher speed, and surveying larger areas with carrying heavier payloads. However, it needs a broader space for takingoff and landing because of its large wings to lift and maintain the system on constant forward motion. Rotary-wing UAV covers the difficulty of fixed-wing UAVs, so it is not restricted to a place for taking-off and landing. Rotary-wing UAV can be considered as a platform for a real-time scenario. We can combine sensors to determine the high performance of drones. A proper combination of camera sensors and UAVs cope with incidents that may occur on the sea. According to Klimkowska (2016), combining the optical camera with thermal imaging camera suggested to detect ships and deliver the meaning of the monitored situation dealing with marine surveillance. This study chooses optical images to process images in real time as preliminary research. In addition, GPS/INS can determine the quality of location accuracy of processed images, and the wireless transmission is the key important role to deliver the acquired data in real time with defining how long it takes to get data from drone to a processing server.

Real-time image processing: The real-time mapping module aims to process the acquired images to generate individual orthophotos and detect various objects. In order to process the images from the UAV system, this part should cover not only image processing functions but also data transmission functions. The image processing functions covers direct georeferencing, individual orthophoto generation and object detection, and more details will be explained in 2.2. The data transmission, on the one hand, covers receiving data from the UAV system and uploading processed results to the visualization part. To implement the data transmission functions, we exploit Flask which is one of pythonbased web frameworks. Specifically, whenever the UAV system sends an image and corresponding GPS/INS data to a certain IP address of the Flask module, the data transmission functions receive the data and parse them to extract values to be used as parameters of the image processing functions. After the given data are processed, the data transmission functions send the individual orthophotos, and corresponding object detection results to the visualization module.

Visualization: After the images are processed, the real-time mapping module uploads the individual orthophoto and corresponding object detection to the visualization module. We implement the visualization module using Mago3D (2018) which is an open-source 3D spatial data visualization platform. Specifically, the visualization module overlays the individual orthophotos and detected objects on a base map and provides the visualization result to public through a web page as shown in Figure 2. In case of ship detection, the visualization module can show detailed attributes of detected ships as shown in Figure 3. It also offers additional functions such as visualizing the flight trajectory and automatic system checking.

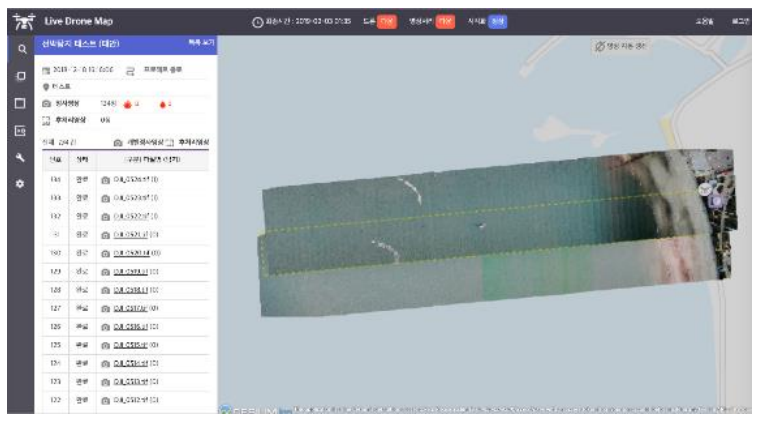

Figure 2. A captured image of orthophotos on Live Drone Map website 


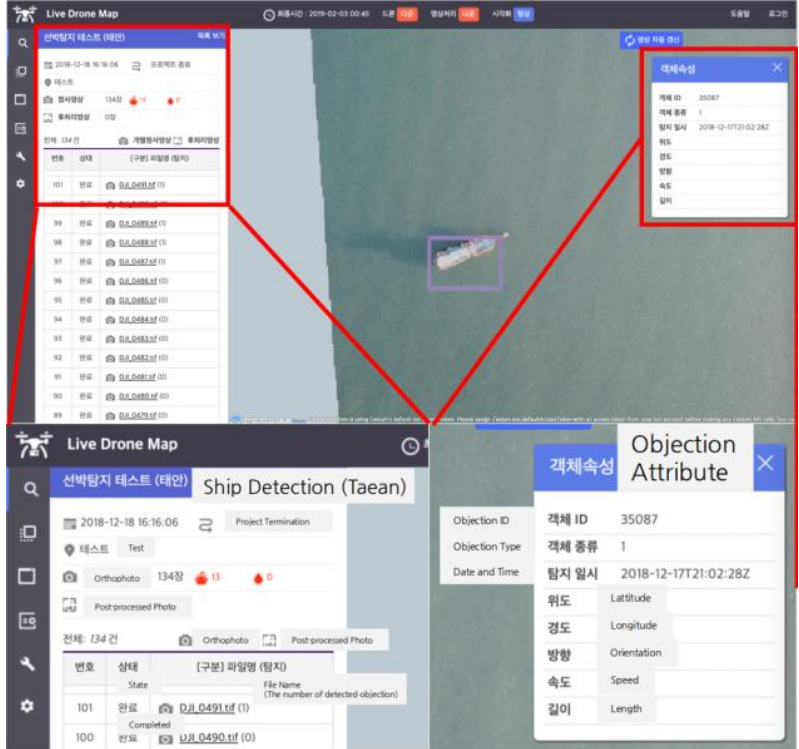

Figure 3. A captured image of detected ship on Live Drone Map website

\subsection{Real-Time Image Processing}

When images and sensor data are input in a processing server, images should be processed as information that is needed in the field. In actual situations, it is believed that the Coast Guard or rescue workers can be dispatched to the site to clean up the situation by quickly receiving information on where the incident is taking place. Implementing the platform in real time demands examining how to proceed accurately and quickly. Real-time processing should meet the acquired images and sensor data to quickly reference the location to the images to generate individual orthophotos and include a faster and more accurate algorithm which automatically detect objects.

A brief description of the mapping and detecting the sea vessel can be found in Figure 4. Images acquired from drones are mapped over 3 steps (Calculating sensor data, Georeferencing, Ortho-Rectification), and, on the one hand, existing spatial information classified into sea and land to detect ships. Finally, the location of the detected vessel can be determined on the orthophotos.

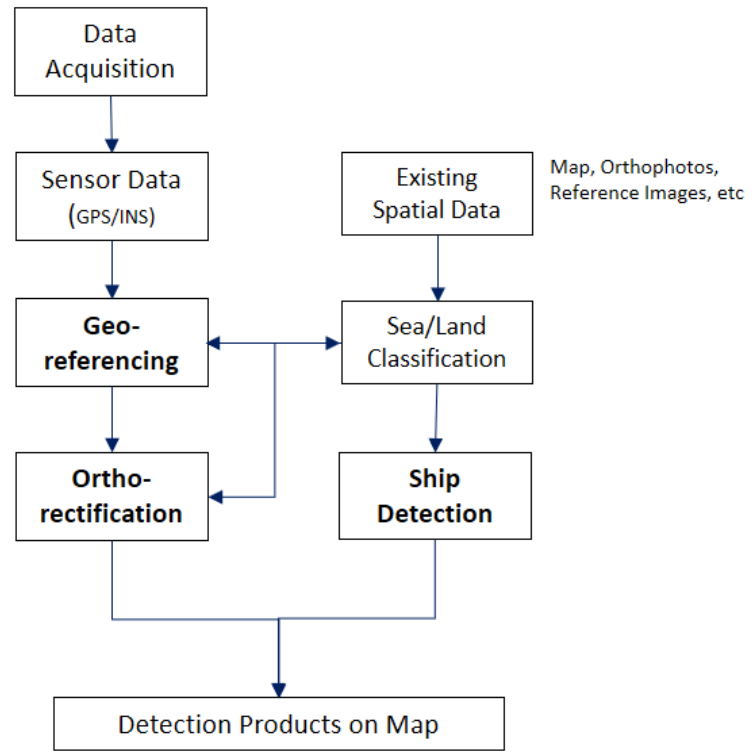

Figure 4. Flowchart of real-time processing
2.2.1 Real-Time Mapping: In order to determine the location of images, it is necessary to generate individual orthophotos through georeferencing process. EO (exterior orientation parameters) representing the location/orientation of the image shall be calculated to generate orthophotos, using pre-processed mounting variables, which can be computed from location/orientation of the drone extracted from the input data. There are two methods of obtaining EO: Direct Georeferencing and Indirect Georeferencing. In SW such as Pix4Dmapper (2011), Photoscan (2010), and OpenDroneMap (2014), which have often been used in terms of higher location accuracy during Indirect Georeferencing, AT (aerial triangulation) have been used mainly. The AT method takes long because it adjusts the EOs of several images at the same time. Direct georeferencing allows rapid processing by multiplying the position/orientation data of the drone, calculated parameters of the mounted sensor (GPS/INS), by a rotation matrix consisting the mounted variable of the sensor, which determines the EO of the image (Cheon, 2017).

Ortho-rectification must be performed in order to generate individual orthophotos from each individual image. Using a method performing ortho-rectifying from determined EOs and DEM (Digital Elevation Model) of images is advantageous for fast transmission because the geometric correction with DEM can enable to rectify with only one image (Kim, 2018). This method calculates the pixel coordinates of the original image, the corner points of the image projected to the DEM defined by the average elevation plane determining the projection range, and the pixel coordinates of the original image through the co-ordination equation for each DEM. After computing pixel values of projected coordinates, texturing is performed by referring to the values of each DEM grid, and generating individual orthophotos where texturing is referenced in two dimensions, which is shown in Figure 5.

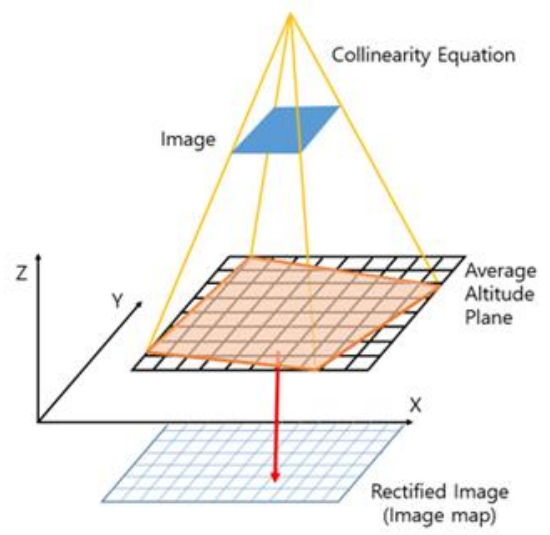

Figure 5. Individual orthophoto generation 
2.2.2 Ship Detection: Ship detection in real-time maritime surveillance should be considered one of the main issues, given the recall that maritime accidents are mainly related to ships. The YOLOv3 algorithm (Redmon et al., 2018) has recently emerged as one of the most used object classifiers. YOLOv3 is a complementary method to the existing YOLO algorithm. In order to detect objects using YOLO algorithm, class prediction, binding box, Feature Pyramid Network (FPN), and feature extractor which is a tool for detecting objects are considered.

YOLOv3 uses independent logistic classifiers to determine whether an object belongs to a particular label or not, during which the classification loss is calculated. The technique simply uses a method of calculating classification loss compared to previous versions of YOLO.

The process in which the tool for extracting objects is formed is flexible and predictable by the size of the object through the grid at the center of the bounding box, so it is simple but still able to detect objects while maintaining fine accuracy. In addition, feature extraction is performed with a hybrid approach to networks used by existing YOLOv2 and DARKNET $\bullet 19$, which provides shortcut connections. In this respect, YOLOv3 can be used as an object detection algorithm in this study, as it was deemed suitable for detecting ships regarded as constant shapes, as well as its advantages of fast processing speed.

\section{EXPERIMENTAL RESULTS}

The platform in this study is intended to monitor and respond quickly to site conditions and should operate in real-time with high accuracy to infer the precise location. The experiment was conducted in order to evaluate 1) the mapping time and the accuracy of mapping 2) detecting time, the accuracy of ship detection, and its location.

\subsection{Data Acquisition}

Dataset: Test was conducted to assess the accuracy and processing time of the mapping to evaluate whether the proposed real-time marine monitoring platform could be applied at the actual site. The experiment was conducted in Tongyeong, Gyeongsangnam-do, and Taean, Chungcheongnam-do, where ships frequently visit for fishing and it is easy to observe and distinguish many different types of ships. The places are suitable for taking-off and landing essential for unmanned aircraft operations. We used a different type of drones and sensors to evaluate the quality of mapping accuracy depending on the used system. 100 images from the collected set were used as a set of data for each test.

Since, in Tongyeong, there are the coast of the island, offshore structures and ships, the images were acquired at different altitudes using the rotary-wing aircraft, which has a relatively long operating distance and time. Because the higher the flight altitude, the wider the ground coverage of individual images, the larger the area observed for high altitude flight missions, and the larger the ground sample distance (GSD), resulting in a difference in location accuracy. The errors obtained at different elevations were identified to compare which elevations had adequate positional accuracy while covering regions we can observe sufficient ships. Tables and images of acquired data in Tongyeong can be found in Table 6, Figure 6 . The resolution of images $6000 \times 4000$.

\begin{tabular}{|c|c|c|}
\hline Altitude & $\begin{array}{c}\text { Ground Sample } \\
\text { Distance (GSD) } \\
\left(\mathrm{X}^{*} \mathrm{Y}\right)(\mathrm{cm})\end{array}$ & $\begin{array}{c}\text { Ground Coverage } \\
(\mathrm{m})\end{array}$ \\
\hline $200 \mathrm{~m}$ & $3.4 \times 3.4$ & $204 \times 136$ \\
\hline $350 \mathrm{~m}$ & $6.0 \times 6.0$ & $358 \times 239$ \\
\hline $500 \mathrm{~m}$ & $8.5 \times 8.5$ & $511 \times 341$ \\
\hline
\end{tabular}

Table 1. Data description in Tongyeong

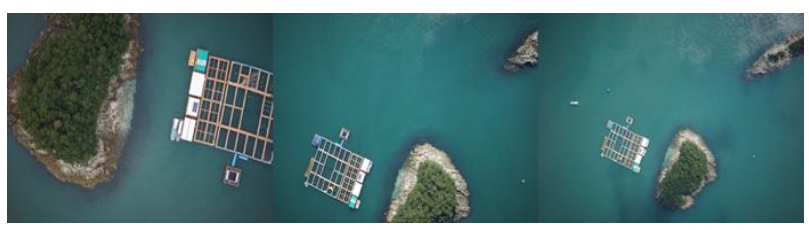

Figure 6. Sample images of dataset in Tongyeong, South Korea

In Taean, tests were carried out near the sandy beach port. In order to show the real-time operation of the system, another rotary-wing UAV was used at an altitude of $200 \mathrm{~m}$ using its own mounted camera. The resolution of images 4000 x 3000. Tables and images of acquired data can be found in Table 2, Figure 7 .

\begin{tabular}{|c|c|c|}
\hline Altitude & $\begin{array}{c}\text { Ground Sample } \\
\text { Distance (GSD) } \\
\left(\mathrm{X}^{*} \mathrm{Y}\right)(\mathrm{cm})\end{array}$ & $\begin{array}{c}\text { Ground Coverage } \\
(\mathrm{m})\end{array}$ \\
\hline $200 \mathrm{~m}$ & 6.67 & $267.1 \times 200.2$ \\
\hline
\end{tabular}

Table 2. Data description in Taean

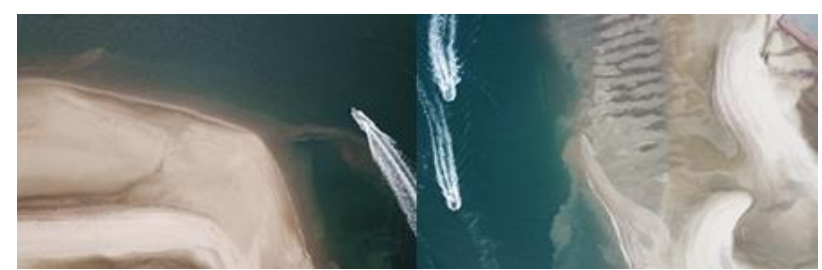

Figure 7. Sample images of dataset in Taean, South Korea

\subsection{Mapping}

In this study, direct georeferencing is performed to send images containing location in real time because the maritime environment makes it difficult to acquire GCPs with accurate location values (Ryu, 2018). Here, the method of direct georeferencing requires the least processing time by calculating EOs by averaging the sensor position/orientation that is put in before and after the images were taken is efficient. If the processing time is less than 10 seconds, the user can make a judgement for an urgent situation. Hence, quantitative criteria for real-time mapping were determined within 10 seconds. 
3.2.1 Mapping Time: While performing real-time monitoring, promptly processing acquired data is essential, and processing time is an important indicator of real-time availability. In order to receive images in real time, it is appropriate to ensure that the wireless transmission is low in jamming and that the drones are well visible. Therefore, data acquired in Taean, Chungcheongnam-do, were used for the image mapping processing time measurement experiment, which is relatively easy to have a place for landings, white sand beaches. After acquiring images from drones, the processing time measurement experiment used to measure an average time needed for users to check the screen within a certain interval after processing them from the processing server. Because measuring time repeatedly with a constant term requires sufficient data, processing time of every 100 images for a dataset was measured. The overall processing time of $3.26 \mathrm{~s}$ was checked on average, shown in Table 3, which corresponds the standard of real-time mapping.

\begin{tabular}{|c|c|c|}
\hline Mapping Time(s) & Dataset1 & Dataset2 \\
\hline Total & $3.30 \pm 0.62$ & $3.24 \pm 0.50$ \\
Mapping Time(s) & & \\
\hline
\end{tabular}

Table 3. Total Mapping Time

3.2.2 Mapping Accuracy: In this study, the accuracy of the location should also be considered, as it should be possible to extract the location of the interested region for an appropriate response, rather than simply monitoring maritime safety. Image mapping accuracy was defined as the minimum accuracy for locating marine monitoring activities in meters as a unit. For errors, we measured the mean square error (RMSE) of the coordinates for the same point of mapping image with the reference points acquired from precise GPS survey. Finally, the location accuracy of the overall image was evaluated.

In Tongyeong area in Figure 8, the reference points have been established with five ground inspection points which are fixed coordinates that allow to obtain GCP easily and three offshore inspection points that can be considered as a reference point in the marine environment.

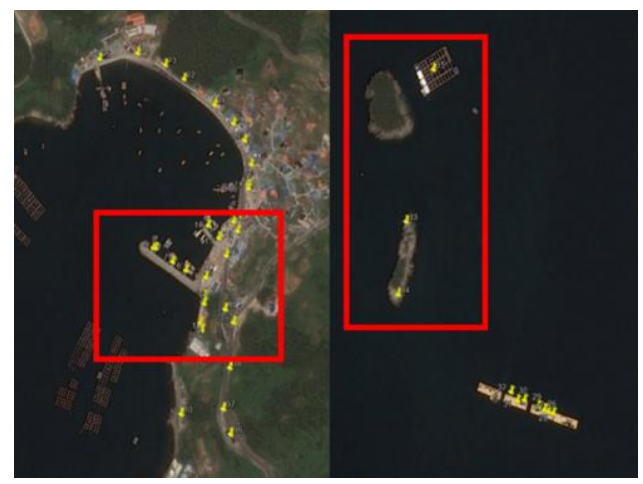

Figure 8. Inspection points in Tongyeong

Absolute accuracy was assessed for each of the five inspection points by altitude. Absolute accuracy of $1.68 \mathrm{~m}, 2.02 \mathrm{~m}$ and 3.85 $\mathrm{m}$ respectively were determined at three different altitudes, in Table 4. Since the size of large ships and ferries can be more than 100 meters, the above error is unlikely to cause a problem that ships are not recognized at the scene. In case of a small ship, the location of the sea vessel can be identified visually within $3 \mathrm{~m}$, which can be considered significant in informing about the location.

\begin{tabular}{|c|c|c|c|c|c|c|c|}
\hline \multirow{2}{*}{$\begin{array}{c}\text { Al- } \\
\text { titude } \\
(\mathrm{m})\end{array}$} & \multicolumn{2}{|c|}{ Average } & \multicolumn{2}{c|}{$\begin{array}{c}\text { Standard } \\
\text { Deviation }\end{array}$} & \multicolumn{2}{|c|}{ RMSE } & \begin{tabular}{c} 
Total \\
RM \\
\cline { 2 - 7 }
\end{tabular} \\
\cline { 2 - 7 } & & $\begin{array}{c}\mathrm{Y} \\
(\mathrm{m})\end{array}$ & $\begin{array}{c}\mathrm{X} \\
(\mathrm{m})\end{array}$ & $\begin{array}{c}\mathrm{Y} \\
(\mathrm{m})\end{array}$ & $\begin{array}{c}\mathrm{X} \\
(\mathrm{m})\end{array}$ & $\begin{array}{c}\mathrm{Y} \\
(\mathrm{m})\end{array}$ & $\begin{array}{c}\text { SE } \\
(\mathrm{m})\end{array}$ \\
\hline 200 & -0.76 & 1.31 & 0.37 & 0.59 & 0.85 & 1.45 & 1.68 \\
\hline 350 & -1.36 & -0.11 & 0.94 & 1.05 & 1.67 & 1.13 & 2.02 \\
\hline 500 & -2.46 & 2.47 & 1.25 & 1.08 & 2.76 & 2.69 & 3.85 \\
\hline
\end{tabular}

Table 4. Mapping Accuracy in Tongyeong

In Taean, 10 grounded-based points in Figure 9 were selected as inspection points to evaluate the mapping absolute accuracy with mean square error.

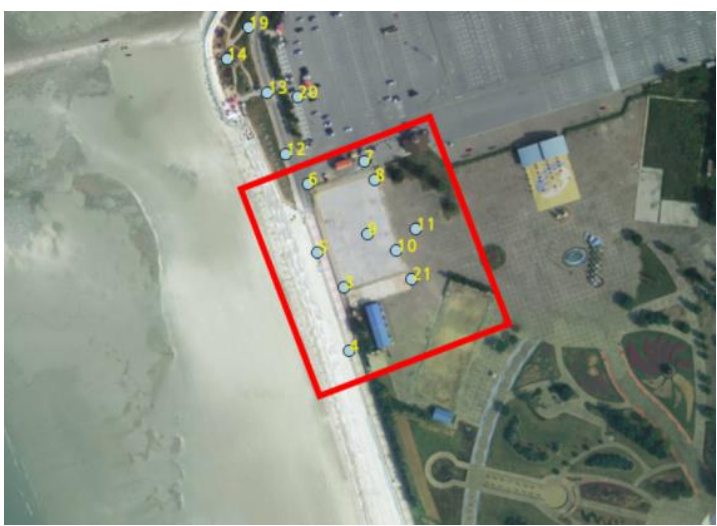

Figure 9. Inspection points in Taean

The RMSE value was checked to be $14.36 \mathrm{~m}$ and the errors differed more than $10 \mathrm{~m}$ compared to the results of the Tongyeong experiment Table 5. Since the drone used in Taean is lower than the accuracy of the drone's GPS/INS sensors used in Tongyeong, the RMSE can be seen as a significant difference. As with the results in Tongyeong, however, considering the size of large ships, ferries, and small ships, the error of about 14 meters may not be considered a major error in informing the ship's location.

\begin{tabular}{|c|c|c|c|c|c|c|c|}
\hline \multirow{2}{*}{$\begin{array}{c}\text { Al- } \\
\text { titude } \\
(\mathrm{m})\end{array}$} & \multicolumn{2}{|c|}{ Average } & \multicolumn{2}{|c|}{$\begin{array}{l}\text { Standard } \\
\text { Deviation }\end{array}$} & \multicolumn{2}{|c|}{ RMSE } & \multirow{2}{*}{\begin{tabular}{|c} 
Total \\
RM \\
SE \\
(m)
\end{tabular}} \\
\hline & $\begin{array}{l}X \\
(\mathrm{~m})\end{array}$ & $\begin{array}{c}\mathrm{Y} \\
(\mathrm{m})\end{array}$ & $\begin{array}{c}X \\
(\mathrm{~m})\end{array}$ & $\begin{array}{c}\mathrm{Y} \\
(\mathrm{m})\end{array}$ & $\begin{array}{c}X \\
(\mathrm{~m})\end{array}$ & $\begin{array}{c}\mathrm{Y} \\
(\mathrm{m})\end{array}$ & \\
\hline 200 & 12.94 & -3.71 & 3.65 & 3.40 & 13.45 & 5.04 & 14.36 \\
\hline
\end{tabular}

Table 5. Mapping Accuracy in Taean 


\subsection{Ship Detection}

3.3.1 Construction of Deep Learning Dataset and Verification of Accuracy: In order to detect a sea vessel in realtime, detection method has to work fast and fully automatically, and these requirements are met by deep learning approaches. To utilize techniques mentioned above, it is necessary to have a dataset with significant number of samples. To test the YOLOv3 method for marine application, we built our own dataset. It contains 1) images acquired from drones which were additionally rotated to increase number of samples; 2) data provided by Kaggle (2018), a machine learning-based data analysis community; 3 ) satellite image data released on the Internet. Ship candidates were extracted from images using BBox-Label-Tool (2017) in Figure 10. Our model was trained with 1701 positive and 2971 negative samples.

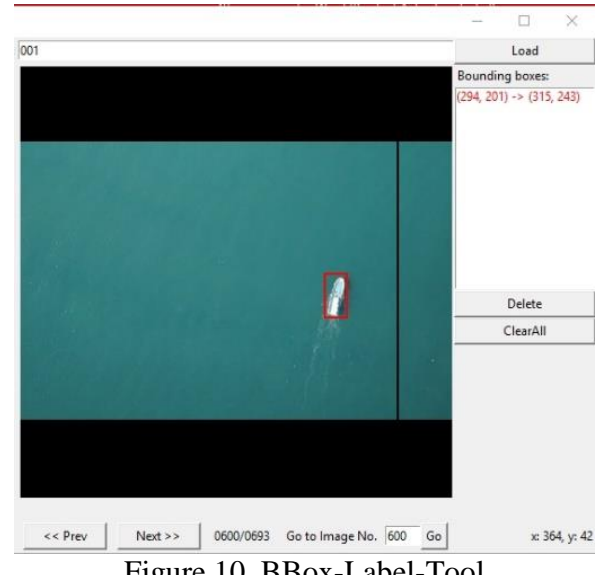

Figure 10. BBox-Label-Tool

The following accuracy has been verified for the set described above and for trained data using YOLO ver.3. mAP (mean average precision) recorded $89.17 \%$ accuracy of 7900 iterations, $90.04 \%$ accuracy of 8300 iterations, and $89.76 \%$ accuracy of 8400 iterations. Through this study, images could be applied to the sea vessel detection algorithm to verify automatic detection of the ship's location in the given Figure 11. The detecting time of the ship detection algorithm corresponded rapid ship detection, for up to a second, which could be accepted to conform to the purpose of this study.

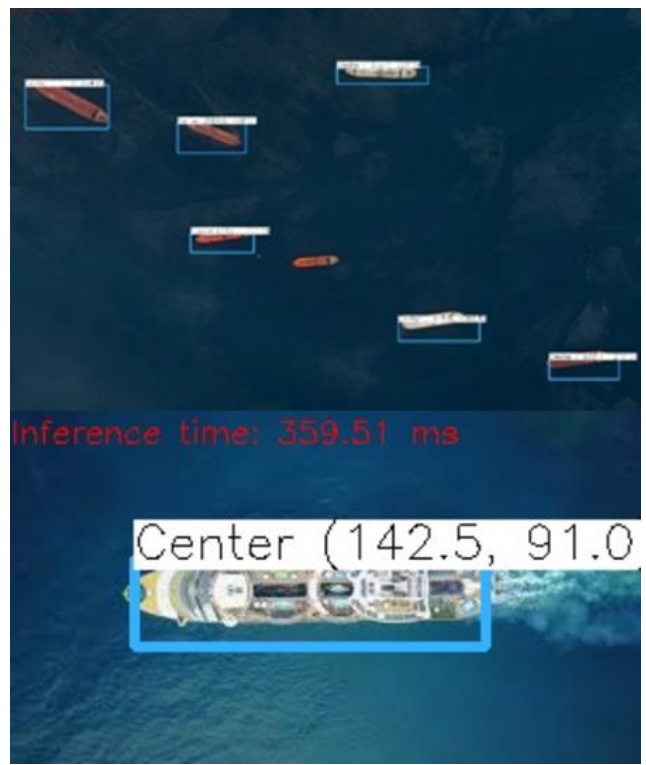

Figure 11. Detected ships by YOLOv3 algorithm
3.3.2 Ship Location Accuracy: GPS acquired on a ship in the field and algorithm calculation results were compared. RMSEs for the location of ships determined at each altitude were $2.47 \mathrm{~m}$, $5.19 \mathrm{~m}, 8.25 \mathrm{~m}$, seen in Table 11. Since the maximum error is about 8 meters, up to an altitude of 500 meters can be considered to be a permitted range for the real-time vessel detection mapping.

\begin{tabular}{|c|c|c|c|c|c|}
\hline \multirow{2}{*}{ Altitude } & \multirow{2}{*}{ Dataset } & \multirow{2}{*}{$\begin{array}{l}\text { The number } \\
\text { of detected } \\
\text { ships }\end{array}$} & \multicolumn{2}{|c|}{ RMSE } & \multirow{2}{*}{$\begin{array}{c}\text { Total } \\
\text { RMSE } \\
(\mathrm{m})\end{array}$} \\
\hline & & & $X(m)$ & $\mathrm{Y}(\mathrm{m})$ & \\
\hline \multirow{2}{*}{$200 \mathrm{~m}$} & A1 & 9 & 0.77 & 0.54 & \multirow{2}{*}{$2.47 \mathrm{~m}$} \\
\hline & A2 & 11 & 2.82 & 1.85 & \\
\hline \multirow{3}{*}{$350 \mathrm{~m}$} & B1 & 10 & 2.74 & 5.34 & \multirow{3}{*}{$5.19 \mathrm{~m}$} \\
\hline & B2 & 16 & 2.50 & 3.38 & \\
\hline & B3 & 24 & 3.33 & 4.33 & \\
\hline \multirow{2}{*}{$500 \mathrm{~m}$} & $\mathrm{C} 1$ & 19 & 1.02 & 4.98 & \multirow{2}{*}{$8.25 \mathrm{~m}$} \\
\hline & $\mathrm{C} 2$ & 12 & 3.79 & 7.58 & \\
\hline
\end{tabular}

Absolute accuracy was assessed for the location of ship detection through datasets acquired in Taean. The results of comparing the results of the ship GPS with the algorithm calculation are shown in the table. RMSEs of $18.4 \mathrm{~m}, 11.7 \mathrm{~m}$ and $18.3 \mathrm{~m}$ were checked for the three sets in Table 12.

\begin{tabular}{|c|c|c|c|c|c|}
\hline \multirow{2}{*}{ Altitude } & \multirow{2}{*}{ Dataset } & \multirow{2}{*}{$\begin{array}{c}\text { Detected } \\
\text { ships }\end{array}$} & \multicolumn{2}{|c|}{ RMSE } & Total \\
\cline { 4 - 5 } & & & $\mathrm{Y}(\mathrm{m})$ & $\begin{array}{c}\text { RMSE } \\
(\mathrm{m})\end{array}$ \\
\hline \multirow{2}{*}{$200 \mathrm{~m}$} & $\mathrm{~A}$ & 4 & 16.3 & 7.1 & 18.4 \\
\cline { 4 - 6 } & $\mathrm{B}$ & 4 & 3.8 & 9.7 & 11.7 \\
\cline { 4 - 5 } & $\mathrm{C}$ & 7 & 14.4 & 6.3 & 18.3 \\
\hline
\end{tabular}

Table 12. Ship Location Accuracy in Taean

The mapping accuracy for the entire set can be found in Table 13 . We can grasp three perspectives at the table. One is the difference between the results in Taean and those in Tongyeong. In Tongyeong, higher mapping accuracy and ship location accuracy were evaluated than in Taean, and it is estimated that the difference was significant depending on sensors on drones influence on the result of mapping because we used more precise GPS/INS sensors in Tongyeong. Second, depending on the altitude at which drone is flying, we can see from the Tongyeong data that errors can be aggravated. Third, the ship's location accuracy may be more weighted than its location accuracy. Nonetheless, overall location accuracy can be evaluated to fit in the standard for this mapping platform because the maximum error is lower than $20 \mathrm{~m}$ within a range that we can watch a sea vessel. 


\begin{tabular}{|c|c|c|c|c|}
\hline Accuracy & $\begin{array}{c}\text { Taean } \\
(200 \mathrm{~m})\end{array}$ & $\begin{array}{c}\text { Tongyeong } \\
(200 \mathrm{~m})\end{array}$ & $\begin{array}{c}\text { Tongyeong } \\
(350 \mathrm{~m})\end{array}$ & $\begin{array}{c}\text { Tongyeong } \\
(500 \mathrm{~m})\end{array}$ \\
\hline $\begin{array}{c}\text { Mapping } \\
\text { Accuracy } \\
\text { (RMSE) }\end{array}$ & $14.36 \mathrm{~m}$ & $1.68 \mathrm{~m}$ & $2.02 \mathrm{~m}$ & $3.85 \mathrm{~m}$ \\
\hline $\begin{array}{c}\text { Ship Location } \\
\text { Accuracy } \\
\text { (RMSE) }\end{array}$ & $16.56 \mathrm{~m}$ & $2.47 \mathrm{~m}$ & $5.19 \mathrm{~m}$ & $8.25 \mathrm{~m}$ \\
\hline
\end{tabular}

Table 13. Overall accuracy

\section{CONCLUSIONS}

This study proposed a platform using UAV for real-time marine monitoring. We focused on providing the location for ships quickly and accurately, as ship-related damage accounts for a large number of possible accidents and damage in the maritime environment. Since information about location matters in terms of marine surveillance, direct georeferencing was performed and individual orthophotos were generated in real-time. Also, the ships were automatically detected to provide their location.

In Taean, the accuracy and real-time image transmission were assessed by mapping it in real-time, which took an average of 3.26 seconds for the information to be viewed as suitable for realtime transmission. The position of the ship taken from $500 \mathrm{~m}$ altitude was determined with absolute accuracy of $8.25 \mathrm{~m}$, which was assessed to be detectable at the site considering the size of the ship.

A model was then established to detect and provide information on the location of the ship, with the time to detect the ship within one second, and the RMSE for the ship detection location recorded $16.56 \mathrm{~m}$ in Taean and $8.25 \mathrm{~m}$ in Tongyeong at $500 \mathrm{~m}$ high. Because of the relatively high mapping accuracy of the data acquired in Tongyeong, a more accurate GPS/INS sensor can determine the location accuracy of the ship, but $16.56 \mathrm{~m}$ RMSE in Taean can also be seen as having no major errors in determining the location of the ship on the site.

This research could be an idea for the implementation of a realtime marine monitoring platform using UAVs applicable at a later at actual site. We expect the platform that could reduce fatal losses from marine accidents by testing on specific scenarios such as accident oil spill, red tide and environmental maintenance that could occur at the site.

\section{ACKNOWLEDGEMENTS}

This research was supported by the project named "UAV-based Marine Surveillance System Development" which is a part of the project named "Wide Integrated Surveillance System of Marine Territory" funded by the Ministry of Maritime Affairs and Fisheries. (1525008596)

\section{REFERENCES}

European Maritime Safety Agency. 2018. Annual Overview of Marine Casualties and Incidents 2018. www.emsa.europa.eu

ALLIANZ GLOBAL CORPORATE \& SPECIALTY. 2018. Safety and Shipping Review 2018: An annual review of trends and developments in shipping losses and safety. www.agcs.allianz.com

K. Lee. 2016. A Study on the Actual Condition and the Countermeasure of Marine Accidents. Korean Association of Police Science, 61, 27-53. http://kiss.kstudy.com
The Ministry of Oceans and Fisheries. 2018. Current Status of Marine Incidents. http://www.index.go.kr/potal/stts/idxMain/selectPoSttsIdxMain Print.do?idx_cd=1770\&board_cd=INDX_001

You, H., Choi, K., Lee, I. 2017. Ship Positioning Using UAV Optic Images and GPS/IMU for Maritime Surveillance. Journal of Korean Society for Geospatial Information Science. 61-62.

Carpenter, A. 2013. Satellites and their Role in EU Maritime Security, Safety and Marine Environmental Protection. Working paper for UACES Annual Conference.

Duan, G. J., \& Zhang, P. F. 2014. Research on Application of UAV for Maritime Supervision. Journal of Shipping and Ocean Engineering, 4, 322-326.

Dimitriou, G. 2013. Integrating Unmanned Aerial Vehicles into surveillance systems in complex maritime environments. NAVAL POSTGRADUATE SCHOOL MONTEREY CA.

Klimkowska, A., Lee, I., \& Choi, K. 2016. Possibilities of UAS for maritime monitoring. The International Archives of Photogrammetry, Remote Sensing and Spatial Information Sciences, 41, 885 .

mago3D. 2018. http://www.mago3d.com/eng/ (29 March 2019)

Pix4D, S. A. 2014. Pix4dmapper. https://www.pix4d.com/ (29 March 2019).

AgiSoft PhotoScan Professional. 2016 http://www.agisoft.com/downloads/installer/ (29 March 2019).

Open Drone $\quad$ Map. 2014 https://github.com/OpenDroneMap/OpenDroneMap (29 March 2019).

Kim, H. 2018. Generation of Individual Ortho-images Based on Reference Images for Facility Inspection. Dept of Geoinformatics, The University of Seoul, Seoul, Republic of Korea.

Cheon, J. 2017. Rapid Image-map Generation for UAV Based Real-time Monitoring. Dept of Geoinformatics, The University of Seoul, Seoul, Republic of Korea.

Kaggle. 2018. https://www.kaggle.com/ (29 March 2019).

Bbox-Label-Tool. 2017. https://github.com/puzzledqs/BBoxLabel-Tool (29 March 2019).

Redmon, J. and Farhadi, A., 2018. Yolov3: An incremental improvement. arXiv preprint arXiv:1804.02767. 